\title{
A comparison of regadenoson and treadmill exercise in myocardial perfusion imaging
}

\begin{abstract}
The aim of this research is to find if the pharmacologic agent regadenoson, will provide more false positives and lower positive predictive value than a treadmill exercise when doing a myocardial perfusion stress test.

Methods: The patients for this research were the first 34 patients that were stressed with a treadmill exercise and the first 34 patients that were stressed using regadenoson. These patients were selected from a local hospital cardiac catheterization lab between the years of 2013 to 2015 starting January 2015, and working backward. These patients had to have a positive myocardial perfusion study and to have a catheterization procedure within six months of the last MPI test. We measured the false positive rate and the positive predictive value for both a treadmill exercise and regadenoson, using the catheterization results as the reference standard.
\end{abstract}

Results: The findings suggest that regadenoson provides a higher false positive rate and a lower positive predictive value than a treadmill exercise.

Conclusion: A treadmill exercise should be more utilized whenever possible to prohibit unnecessary cardiac catheterization procedures.

Keywords: patients, regadenoson, dipyridamole, dobutamine, adenosine

\author{
Volume 5 Issue I - 2018
}

\author{
Hani Mousa Alkhalaf, Ghadeer Alkhalaf, ${ }^{2}$ \\ Dawn Burkhardt, ${ }^{3}$ Cybil J Nielsen, ${ }^{2}$ Elisabeth \\ von der Lohe ${ }^{4}$ \\ 'King Faisal University, Saudi Arabia \\ Indiana University School of Medicine, USA \\ ${ }^{3}$ Indiana University Hospital, USA \\ ${ }^{4}$ Indiana University School of Medicine, Interventional \\ Cardiology and Interventional Fellowship Program, USA
}

Correspondence: Hani Mousa Alkhalaf, King Faisal University (student at college of medicine), Saudi Arabia,

Email medicine-14|4@hotmail.com

Received: December 31, 2017 | Published: January 25, 2018
Abbreviations: MPI, myocardial perfusion imaging; IRB, institutional review board; HIPPAA, health insurance portability and accountability act

\section{Introduction}

Myocardial perfusion imaging (MPI) is a noninvasive procedure used to evaluate coronary artery disease through the distribution of the radioactive tracer to the myocardium. Areas of decreased or diminished uptake of the radioactive tracer are suspicious for blockage. ${ }^{1}$ Patients who have a positive MPI result are often advised to have a cardiac catheterization procedure to have further assessment of the coronary arteries blockage. A cardiac catheterization procedure determines what percentage the artery is blocked. Patients with $50 \%$ stenosis or greater are considered positive in the catheterization lab. ${ }^{2}$ Myocardial perfusion study can be performed with either a treadmill exercise or the pharmacologic stress agent regadenoson.

A myocardial perfusion stress test with a treadmill exercise is the preferred approach over the pharmacologic stress agent regadenoson to stress patients because it provides additional diagnostic and prognostic information. ${ }^{2}$ For a treadmill exercise stress test to be valid, patients have to achieve their target heart rate. ${ }^{3}$

However, because most patients undergoing a myocardial perfusion stress test are unable to walk a treadmill or achieve their target heart rate, pharmacologic stress agents, such as regadenoson provide an alternative way to stress those patients. Moreover, the use of these agents simplifies the nuclear medicine staffs' work that leads to better efficiency in the nuclear medicine departments. In addition to that, regadenoson has lower incidence of side effects than other pharmacologic stress agents which leads to better patient satisfaction. ${ }^{4}$

Therefore, the pharmacologic stress agent regadenoson is more frequently used to stress MPI patients. There are several pharmacologic stress agents such as dipyridamole, dobutamine, adenosine, and regadenoson. Among all these pharmacologic stress agents, regadenoson was compared to the treadmill exercise stress test because regadenoson is the first $\mathrm{A}_{2 \mathrm{~A}}$ receptor agonist approved for clinical use. ${ }^{4}$

The results of MPI influence the type of treatment patients might have which includes a cardiac catheterization procedure. It is critical to prevent unnecessary cardiac catheterization procedures to have lower cost, lower mortality, and lower morbidity. The purpose of this research was to assess the effectiveness of the pharmacologic stress agent regadenoson versus the treadmill exercise by measuring their false positive rates and positive predictive values using the cardiac catheterization as the reference standard.

\section{Materials and methods}

Institutional Review Board (IRB) approval with a HIPAA waiver of authorization was obtained prior to collecting data. This retrospective study was done to compare the treadmill exercise and the pharmacologic stress agent regadenoson in providing a myocardial perfusion rest/stress test. The patients for this research were collected between the years of 2013 to 2015 starting January 2015 working backward. The patients for this research were selected if they had tested positive for a myocardial perfusion study and had a cardiac catheterization procedure within six months of having a positive MPI study. Patients who had a negative MPI study were not included even if they had a cardiac catheterization procedure. There were not so many patients with a negative MPI that undergone a cardiac catheterization procedures. The first 34 treadmill patients and the first 34 regadenoson patients who fit this criteria were selected. The collection of the data was retrospective.

The patients were categorized as positive or negative in the cardiac 
catheterization lab based on the percentage of stenosis present in their coronary arteries. The false positive rate and the positive predictive value for both the treadmill exercise and the regadenoson stress were calculated and compared.

\section{Results}

The results were identified for 34 patients that had a cardiac catheterization procedure after a positive myocardial perfusion stress test with regadenoson and for 34 patients who had a positive myocardial perfusion stress test with a treadmill. The false positive rate for patients who did the treadmill exercise was $24 \%$. The false positive rate for patients that were stressed with regadenoson was $35 \%$. In addition, the positive predictive value for patients that did a treadmill was $77 \%$. The positive predictive value for patients that were stressed with regadenoson was $65 \%$ (Table 1 ).

Table I A comparison of stress test results by the type of stress

\begin{tabular}{lll}
\hline Type of stress test & Treadmill exercise & Regadenoson \\
\hline False positive rate & $8 / 34(24 \%)$ & $12 / 34(35 \%)$ \\
Positive predictive value & $26 / 34(77 \%)$ & $22 / 34(65 \%)$ \\
\hline
\end{tabular}

\section{Discussion}

The patients' data was analyzed using positive predictive values and false positive rates for both a treadmill exercise and regadenoson. False positive rates and positive predictive values are terms used to express the accuracy of MPI results. In reference to the data table, the false positive rate for regadenoson is greater than the false positive rate for a treadmill stress test. Furthermore, the positive predictive value for a treadmill stress test is higher than the positive predictive value for regadenoson.

Because the treadmill exercise provides a lower false positive rate and a greater positive predictive value, it remains the preferred method to stress MPI patients whenever possible. Previous research has found that a treadmill exercise stress test is preferable to all the pharmacologic stress agents including regadenoson, which supports this research finding. ${ }^{2}$ Some limitations to this study include that the patients' age, sex, and medical condition are not defined for both the treadmill and the regadenoson. Another limitation to this study is that the sample size for both types of stress is small.
With the greater false positive rate with utilizing regadenoson for the MPI stress test, patients are likely to undergo a cardiac catheterization procedure. Therefore, regadenoson's use should be limited to those who absolutely cannot do treadmill. Further research should be done to identify the sensitivity and specificity for both the treadmill exercise and regadenoson.,

\section{Conclusion}

Regadenoson provides a higher false positive rate and a lower positive predictive value than a treadmill exercise. Consequently, there should be more utilization of treadmill exercise whenever possible when stressing patients for MPI to prevent an unnecessary cardiac catheterization procedures to limit the numbers of patients having cardiac catheterization procedures.

\section{Acknowledgements}

None.

\section{Conflict of interest}

Author declares that there is no conflict of interest.

\section{References}

1. Arumugam P, Harbinson M, Reyes E, et al. Procedure guidelines for radionuclide myocardial perfusion imaging with single-photon emission computed tomography. Nucl Med Commun. 2013;34(8):813-826.

2. Bires AM, Lawson D, Wasser TE, et al. Comparison of Bruce Treadmill Exercise Test Protocols: Is Ramped Bruce Equal or Superior to Standard Bruce in Producing Clinically Valid Studies for Patients Presenting for Evaluation of Cardiac Ischemia or Arrhythmia with Body Mass Index Equal to or Greater Than 30? J Nucl Med Technol. 2013;41(4):274-278.

3. Botvinick EH. Current Methods of Pharmacologic Stress Testing and the Potential Advantages of New Agents. J Nucl Med Technol. 2009;37(1):14-25.

4. Miller TD, Askew JW, Anavekar NS. Noninvasive stress testing for coronary artery disease. Cardiology Clinics. 2014;32(3):387-404.

5. Parker MW, Morales DC, Slim HB, et al. A strategy of symptom-limited exercise with regadenoson-as-needed for stress myocardial perfusion imaging: a randomized controlled trial. JNucl Cardiol. 2013;20(2):185-196.

6. Johnson SG, Peters S. Advances in Pharmacologic Stress Agents: Focus on Regadenoson. J Nucl Med Technol. 2010;38(3):163-171. 